\begin{tabular}{|c|l|}
\hline Title & Growth of GaA s/A IGaA s hexagonal pillars on GaA s (111)B surfaces by selective area MOV PE \\
\hline Author(s) & Motohisa, J.; Takeda, J.; Inari, M.; Noborisaka, J.; Fukui, T. \\
\hline Citation & $\begin{array}{l}\text { Physica E: Low-dimensional Systems and Nanostructures, 23(3-4), 298-304 } \\
\text { https://doi.org/10.1016/.physe.2003.11.279 }\end{array}$ \\
\hline Issue Date & 200407 \\
\hline Doc URL & http://hdl.handle.net/2115/5526 \\
\hline Type & article (author version) \\
\hline File Information & PE23(3-4).pdf \\
\hline
\end{tabular}

Instructions for use 


\title{
Growth of GaAs/AIGaAs Hexagonal Pillars on GaAs (111)B Surfaces by Selective-Area MOVPE
}

\author{
J. Motohisa *, J. Takeda, M. Inari, J. Noborisaka, T. Fukui \\ Research Center for Integrated Quantum Electronics, Hokkaido University, North 13, \\ West 8, Sapporo 060-8628, Japan
}

\begin{abstract}
We report on the growth of GaAs and GaAs/AlGaAs heterostructured hexagonal pillar structures using selective area (SA) metalorganic vapor phase epitaxy (MOVPE). By doing growth on $\mathrm{SiO}_{2}$ masked (111)B GaAs substrates with circular or hexagonal hole openings, extremely uniform array of hexagonal GaAs/AlGaAs pillars consisting $\{110\}$ vertical facets with their diameter of order of $100 \mathrm{~nm}$ were obtained. Unexpectingly strong intense light emission was observed for the room temperature photoluminescence measurement of the pillar arrays in triangular lattice, which is promising for the application to the photonic crystals to enhance the light extraction efficiency from the materials with high refractive index. Furthermore, it was also found that hexagonal pillars with size $60 \mathrm{~nm}$ and large aspect ratio $(>100)$ by reducing the size of initial hole size of mask, opening a possibility to grow nanowires using epitaxial growth. (This article is published in Physica E, 23, 3-4, pp. 298-304 (2004).)
\end{abstract}

Key words: metalorganic vapor phase epitaxy, selective area growth, photonic crystals, nanowires

PACS:

\section{Introduction}

Selective area (SA) growth on masked substrates is one of the most versatile methods to form semiconductor quantum nanostructures free from process induced damages in a self-organized fashion. Formation of various kind of nanostructures have been reported on SA growth both on (001) and

* Corresponding author.

Email address:

motohisalrciqe.hokudai.ac.jp (J.

Motohisa).
(111)B substrates using metalorganic vapor phase epitaxy (MOPVE). In the case of SA-MOVPE growth on (111)B GaAs, nanostructures surrounded by vertical or inclined $\{110\}$ facets are formed depending on the growth conditions, which makes this approach attractive and powerful to form quantum dots[1], hexagonal structures[2], and Kagome-lattice structures[3] Recently, we have reported on the SA-MOVPE growth on (111)B substrates for the applications to the photonic crystals ( $\mathrm{PhCs}$ ), which are the periodic structure of dielectric materials with their period (or lattice constant) 
$a$ close to the light wavelength. Formation of the air-hole array of GaAs[4,5], and pillar arrays of InGaAs[6] and InP[7] with $a$ down to $0.4 \mu \mathrm{m}$ have been reported. This is motivated by an early study by Hamano et al.[8], where they grew hexagonal pillar arrays of GaAs on masked (111)B substrate with hexagonal opening areas. However, their results were not very sufficient because of the large non-uniformity of the size and shape of the pillars. Furthermore, almost no optical properties of $\mathrm{PhCs}$ fabricated by SA-MOVPE have been reported to date in spite of an expectation that no process induced damage could be introduced. This is partly because the influence of nonradiative surface recombinations becomes significant particularly in GaAs-based materials as the shrinkage of the structural size.

On the other hand, semiconductor nanowires are attracting interest for building blocks of the nanoscale electronics[9] and photonics[10,11] in the bottom-up approach. Nanowires of various materials have been reported, and, especially, realization of heterostructured nanowires are very attractive for the application[12-14]. To now, most of such nanowires are formed by catalyst-assisted vapor-liquid-phase (VLS) growth[15]. We come up to an idea that the growth of hexagonal pillars on (111)B masked substrates can also be used to realize semiconductor nanowires as well as PhCs. If the epitaxial growth is adopted for nanowire fabrication, we can expect much better controllability for the crystallographic structures, crystalline quality, doping, and even heterostructures as well as their precise positioning.

In this paper, we report the formation of GaAs/AlGaAs hexagonal pillar structures by SA-MOVPE on (111)B GaAs substrates for the application of both $\mathrm{PhCs}$ and nanowires.
We will describe the formation of uniform pillar arrays and their photoluminescence (PL) characteristics for PhCs which are important for practical application. Simultaneously, growth of pillars with small diameter and high aspect ratio is attempted for the nanowire application.

\section{Experimental Procedure}

SA-MOVPE growth was carried out in a horizontal, low-pressure system working at $0.1 \mathrm{~atm}$. Trimethylgallium (TMGa), trimethylaluminum (TMAl) and arsine $\left(\mathrm{AsH}_{3}\right)$ were used as source materials. Typical partial pressures were $2.7 \times 10^{-6} \mathrm{~atm}$ for TMGa for GaAs growth, and $5.2 \times 10^{-7}$ and $5.7 \times 10^{-8}$ for TMGa and TMAl, respectively, for $\mathrm{Al}_{0.25} \mathrm{Ga}_{0.75} \mathrm{As}$ growth. Partial pressure of $\mathrm{AsH}_{3}$ was changed from $2.5 \times 10^{-5}$ atm to $5 \times 10^{-4}$ atm. The growth temperatures were also changed from $700^{\circ} \mathrm{C}$ to $850^{\circ} \mathrm{C}$. With these conditions, the growth rate on planar (001) substrate is $0.16 \mathrm{~nm} / \mathrm{sec}$ for $\mathrm{GaAs}$ and $0.04 \mathrm{~nm} / \mathrm{sec}$ for $\mathrm{Al}_{x} \mathrm{Ga}_{1-x} \mathrm{As}$, while the those on both (111)B masked and reference planar substrates depend strongly on the growth conditions. The growth rate for the masked substrates also depends on the size and pitch of the mask patterns.

Figure 1(a) shows a schematic illustration of a $\mathrm{SiO}_{2}$ masked substrate. After the sputtering of $\mathrm{SiO}_{2}$ onto (111)B GaAs substrates, hexagonal holes with size $d_{0}$ were defined by electron beam (EB) lithography and wet chemical etching of $\mathrm{SiO}_{2}$. The holes were arranged to form triangular lattice with a lattice constant (period) $a$. The lattice constant $a$ was varied from $0.2 \mu \mathrm{m}$ to $3 \mu \mathrm{m}$. As we will show below, the size of the initial hole opening give rise to a large influence on the growth of hexagonal pillars. We varied the 
size $d_{0}$ by changing intentionally the EB dose during the lithography, as well as changing the designed hole size. We note that $d_{0}$ is not constant and depends on $a$ due to the proximity effect, even if the EB dose is the same. At the same time, the shape of the hole is circular rather than hexagonal. This was due to the resolution limit in the lithography and etching process. As a result, the diameter $d_{0}$ on the fabricated masked substrates was ranged from about $50 \mathrm{~nm}$ to about $150 \mathrm{~nm}$. With all such complication and deterioration of the shape of the mask pattern, we were able to obtain six-fold symmetric pillars and their uniform and high-density arrays owing to the evolution of sidewall $\{110\}$ facets during the growth[6,8]. (a)

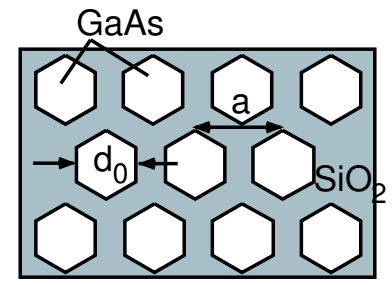

(b)

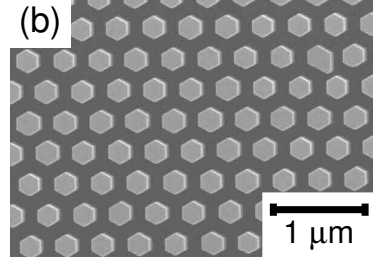

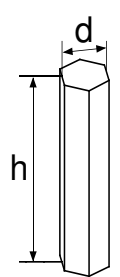

(c)

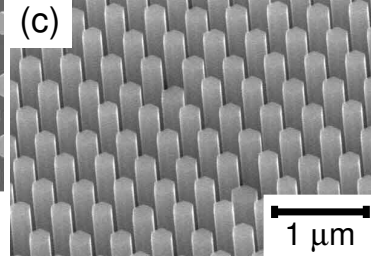

Fig. 1. (a) Schematic illustration of the mask pattern. Typical SEM images of GaAs hexagonal pillar arrays with $a=0.4 \mu \mathrm{m}$ taken from the top (b) and from the oblique angle (c).

Structural characterization was carried out using a HITACHI S-4100 secondary electron microscope (SEM). Photoluminescence was measured at room temperature using $\times 20$ microscope objectives with numerical aperture of 0.4 both for focusing and collection of the light normal to the surface. $\mathrm{Ar}^{+}$laser operating at $514.5 \mathrm{~nm}$ and liquid-nitrogen cooled charge coupled device combined with spectrometer were used for excitation and detection, respectively.

\section{Results and Discussions}

\subsection{Growth of GaAs pillar arrays}

Figures 1(b) and (c) respectively shows typical top view and bird's eye view SEM images of the GaAs pillar arrays of $a=0.4 \mu \mathrm{m}$. The growth temperature $T_{\mathrm{G}}$ was $750^{\circ} \mathrm{C}$ and the $\mathrm{AsH}_{3}$ partial pressure $\left[\mathrm{AsH}_{3}\right]$ is $5 \times 10^{-4} \mathrm{~atm}$. We can see uniform arrays of vertical hexagonal pillars consisting of six $\{110\}$ sidewall facets. As we have mentioned in the previous section, the initial mask opening is circular and the pillar shape does not reflect the pattern shape. This is because slowly growing surfaces appear as facets in SA-MOVPE, and they are $\{110\}$ surfaces in the present growth conditions[2]. Furthremore, due to the symmetry of (111)B, there are 6 vertical $\{110\}$ surfaces, which gives rise to the formation of six-fold symmetric pillars. The average lateral size $d$ of the pillars was $0.24 \mu \mathrm{m}$, and their height $h$ was about $1.4 \mu \mathrm{m}$ for this sample. Similar pillar arrays are obtained when $\left[\mathrm{AsH}_{3}\right]$ is lowered to $2.5 \times 10^{-4} \mathrm{~atm}$. However, further reduction of $\left[\mathrm{AsH}_{3}\right]$ seems to result in the loss of uniformity in the pillar size. When the growth temperature is raised to $850^{\circ} \mathrm{C}$ at $\left[\mathrm{AsH}_{3}\right]=2.5 \times 10^{-4} \mathrm{~atm}$, the growth becomes inhomogeneous and pillar size, both diameter and height, becomes random when the growth temperature is raised to $850^{\circ} \mathrm{C}$. This is consistent with our previous results[5] and it is considered to be due to reevaporation and inhomogeneous nucleation of GaAs. Therefore, the results indicate that the re-evaporation is reasonably suppressed and by lowering the growth temperature. However, when $T_{\mathrm{G}}$ is further lowered to $700^{\circ} \mathrm{C}$, it was not able to obtain uniform pillar arrays due to the lateral overgrowth on $\{110\}$ surfaces becomes prominent. 
It is noted that the pillar height is much larger than the growth thickness $(0.19 \mu \mathrm{m})$ on reference (001) GaAs substrate grown simultaneously in the same run. Around these optimum growth conditions $\left(T_{\mathrm{G}}=750^{\circ} \mathrm{C}\right.$ and $\left.\left[\mathrm{AsH}_{3}\right]=2.5 \sim 5 \times 10^{-4} \mathrm{~atm}\right)$, the enhancement is typically in the order of 10 . This value was found to dependent on the initial size $d_{0}$ of the mask opening, and it becomes larger if $d_{0}$ is smaller. This is consistent with the pervious results by Hamano et al. [8], and is ascribed to the diffusion of growth species from masked areas. The same effect was observed in InGaAs pillar arrays[6]. Furthermore, we also have found that the pillar height becomes higher if $\left[\mathrm{AsH}_{3}\right]$ is reduced, which is explained by $\left[\mathrm{AsH}_{3}\right]$ dependence of growth rate of GaAs on (111)B. More quantitative discussion on the dependence of growth process and pillar size on the growth conditions and mask opening will be described elsewhere[16].

In order to show the uniformity of the pillars quantitatively, we measured the distribution of the pillar size from top view SEM images. The results are shown in Figs.2(a) and (b) for $a=0.6 \mu \mathrm{m}$ and $a=0.4 \mu \mathrm{m}$, respectively. The solid curve in each figure indicates the fitting with gaussian distribution of standard deviation $\sigma$. From these results, the size uniformity $\sigma / d$ of the pillars is measured to be $1.6 \%$ for $a=0.6 \mu \mathrm{m}$, and $2.9 \%$ for $a=0.4 \mu \mathrm{m}$, demonstrating excellent size uniformity of the hexagonal pillars. It seems the tail of the distribution for $a=0.4 \mu \mathrm{m}$ is slightly large. We believe that it is originating from the initial size nonuniformity of the mask pattern since proximity effect is more significant for shoter periods, and could be improoved by the refinement of the EB lithography, At the same time, the uniformity $\sigma / a$ of the pillars with respect to the period $a$, which is an important factor for $\mathrm{PhCs}$, is $1.6 \%$ and $1.7 \%$ for $a=0.6$ and $0.4 \mu \mathrm{m}$, respectively. These values are well below the allowable size fluctuation of for $\mathrm{PhCs}(<5 \%)$, thus our pillar arrays satisfy the requirements in the size uniformity of PhCs.
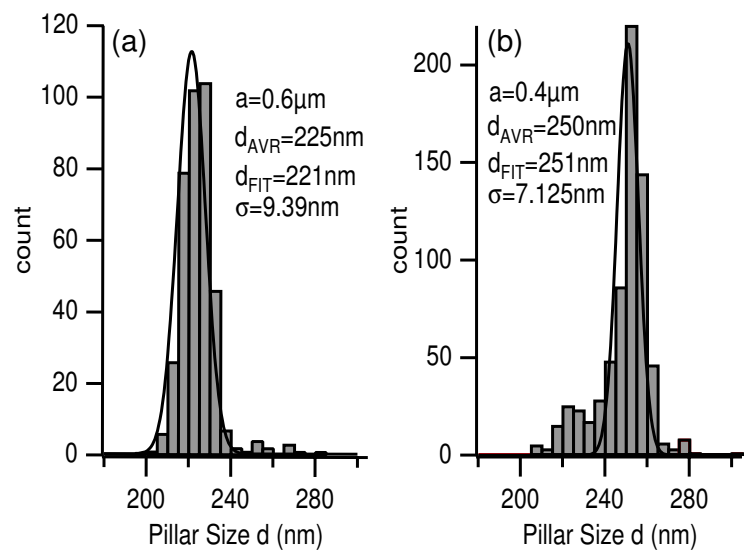

Fig. 2. Histogram of the size distribution of the pillars for $a=0.6 \mu \mathrm{m}$ (a) and $a=0.4 \mu \mathrm{m} \mathrm{(b)}$

\subsection{Growth of AlGaAs/GaAs Heterostruc- ture Pillars and their Photolumines- cence Characterization}

We also carried out SA-MOVPE of GaAs/ $\mathrm{Al}_{0.25} \mathrm{Ga}_{0.75} \mathrm{As}$ heterostructured pillar arrays on (111)B substrates. The layer structure of the heterostructure is shown in Fig. 3(a) together with the designed thickness on (001) substrate. We found that $\mathrm{AlGaAs}$ did not grow selectively at $750^{\circ} \mathrm{C}$, but it was successful at $850^{\circ} \mathrm{C}$. For this reason, the buffer GaAs layer was grown at $750{ }^{\circ} \mathrm{C}$ and the temperature is raised to $850{ }^{\circ} \mathrm{C}$ for overlayers. Furthermore, we reduced the growth rate of the overlayers to by factor of 4 as compared to GaAs buffer $(0.04 \mathrm{~nm} / \mathrm{sec}$ on planar), because the PL was not strong enough if the growth rate was unchanged. The reason for the degradation of PL is not clear at present. Furthermore, it was confirmed that GaAs grows uniformly on AlGaAs layers even at $850^{\circ} \mathrm{C}$ (not shown here), which is inconsistent with the results for growth on GaAs as 
describe above and reported previously[5]. This is not clear either, but one of the reason for this discrepancy is thought to be in the difference of initial nucleation of GaAs on between GaAs and AlGaAs surfaces. Since the growth rate is very high, it gives a significant difference for the growth. More systematic study on the heterostructured pillars are left for future study.

Next, we measured the photoluminescence (PL) of the pillar arrays of the sample shown in Fig. 3(a). The room-temperature PL spectra of the pillar arrays with various $a$ are summarized in Fig. 3(b). Although the pillar size $d$ is somewhat dependent on $a$, it is in the range of $213 \sim 223 \mathrm{~nm}$. Strong emission was observed in each PL spectrum at around $860 \mathrm{~nm}$ close to the band edge of GaAs emission, which is attributable to the emission form GaAs quantum well (QW) formed top on the pillars. The integrated intensity was about 5 to 10 times stronger than that of a semi-insulating GaAs substrate. This emission is surprising considering the small diameter of the pillars, where influence of the non-radiative recombination at the surface in GaAs-based materials is thought to be significant. The PL spectra of reference QW grown on the planar (001) or (111)B substrate is thought to be dominated by the deep levels.

To discuss the behavior of PL intensity on the structural parameters, we discuss about two factors, namely, surface recombination and effect originating form $\mathrm{PhC}$, both of which affects greatly on the emission properties. First of all, the surface recombination is known to considerably reduce the internal emission efficiency. The problem is very significant in GaAs/AlGaAs because of the high density surface states. To qualitatively estimate the effect of surface nonradiative recombination of the pillar sidewall, we solve

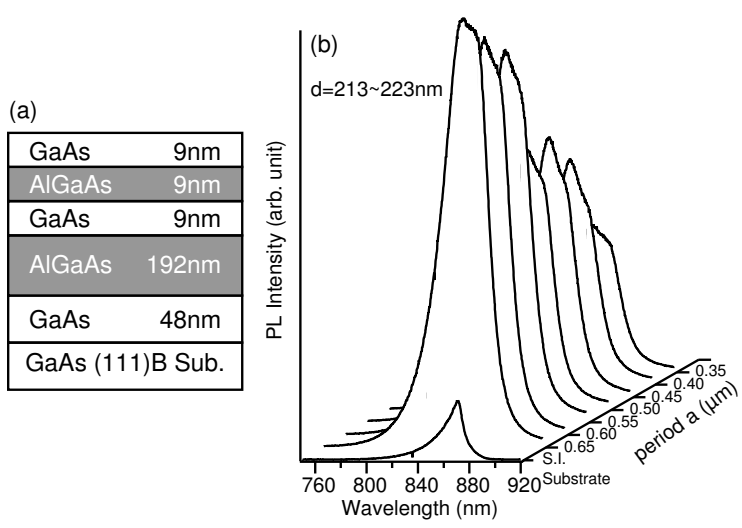

Fig. 3. (a) Layer structure of $\mathrm{AlGaAs} / \mathrm{GaAs}$ heterostructured pillars for the sample for PL measurement. The thickness is for the planar substrate and is much thicker for masked substrates. (b) PL spectra of the hexagonal pillar arrays with different period $a$ of the triangular lattice.

the diffusion equation

$\frac{\partial N}{\partial t}=\nabla(D \nabla N)-\frac{N}{\tau}+g$,

under the boundary condition

$D \frac{\partial N}{\partial n}=v_{s} N$

where $N$ is the density of electron-hole pairs, $D$ the diffusion coefficient, $\tau$ the radiative recombination lifetime, and $g$ the generation rate of the electron-hole pairs, and $v_{s}$ the surface recombination velocity. For simplicity, the pillars are approximated by circular disk (radius $R$ ) haveing radial symmetry, and PL intensity is assumed to be given by $I(R)=2 \pi \int_{0}^{R} N(r) r d r$, Figure 4 shows the normalized PL intensity $I(R) / I(\infty)$, plotted as a function $R$. Two curves represent the results using parameter sets (a) $D=16 \mathrm{~cm}^{2} / \mathrm{sec}, \quad L_{D}(=\sqrt{D \tau})=2 \mu \mathrm{m}$, $v_{s}=7 \times 10^{6} \mathrm{~cm} / \mathrm{sec}$, and (b) $D=16 \mathrm{~cm}^{2} / \mathrm{sec}$, $L_{D}=1.5 \mu \mathrm{m}, v_{s}=1 \times 10^{6} \mathrm{~cm} / \mathrm{sec}$, which are depicted from the reference [17] and respectively corresponds to the low and strong 
excitation regime. Because the diameter of our pillars are about $d=200 \mathrm{~nm}$, the calculation indicates that the intensity is the order of $1 / 500$ or $1 / 1000$ as compared to the bulk structure. In the present experiment, such significant reduction of photoluminescence efficiency seems to be absent. We think this is because AlGaAs layer caps over the sidewall of pillars during the growth (lateral overgrowth), and bare sidewall surface of GaAs QW is not directly exposed to air. Such lateral overgrowth is possible because the lateral growth of AlGaAs on $\{110\}$ critically depends on the growth temperatures and growth rates[18,19]. In fact, the PL intensity of GaAs pillars (without AlGaAs) is not so strong as that of heterostructured pillars. Quantitatively, top barrier thickness around $10 \mathrm{~nm}$ is required to supress the reduction of PL efficiency in the case of near surface quantum wells[20,21]. It is still to be investigated if such thick layer of GaAs and AlGaAs is actually grown on $\{110\}$ sidewalls in the present growth conditions.

Next, we discuss about the effect originating from PhCs. Fan et al.[22] have shown that the light extraction efficiency from slab structures can be enhanced drastically in PhCs as compared to the slab structures. This is because the slabs having large refractive index should exhibit strong internal reflection at the surface, which gives rise to the propagating mode in the slab, and eventually, poor light extraction from the surface. It can be eliminated, however, by fabricating periodic structure on the surface as the coupling to the leaky (radiation) mode is promoted by diffraction or by the photonic bandgap which prohibits lateral propagation. Some experimental demonstrations have already been reported[23-26]. In the results shown in Fig.3(b), the PL intensity increases as $a$. It cannot be explained by the difference of surface recombination since we have

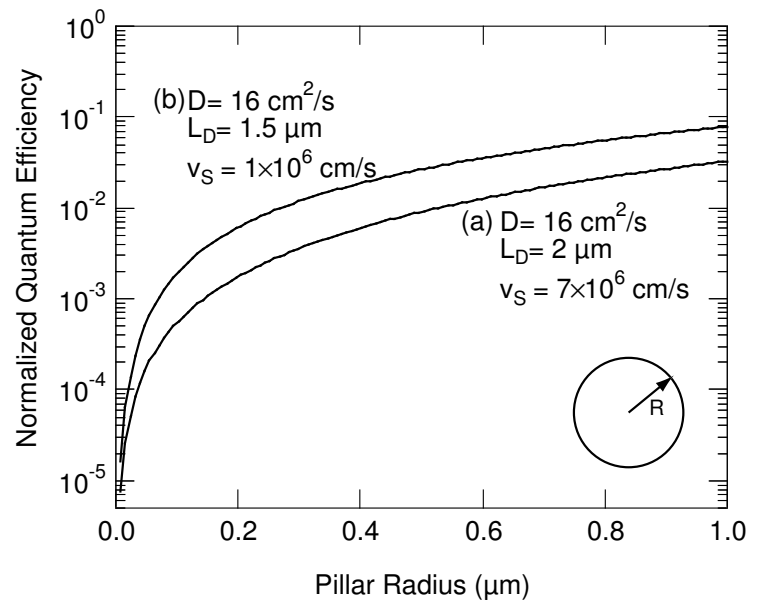

Fig. 4. Effect of surface nonradiative recombination on the photoluminescence efficiency in pillar structures. The parameters for the calculation is (a) $D=16 \mathrm{~cm}^{2} / \mathrm{sec}, L_{D}=2 \mu \mathrm{m}$, $v_{s}=7 \times 10^{6} \mathrm{~cm} / \mathrm{sec}$, and (b) $D=16 \mathrm{~cm}^{2} / \mathrm{sec}$, $L_{D}=1.5 \mu \mathrm{m}, v_{s}=1 \times 10^{6} \mathrm{~cm} / \mathrm{sec}$, which roughly corresponds to the weak and strong excitation regime.

larger pillars for shorter $a$. On the other hand, at fixed wavelenth $\lambda$, the normalized frequency, $v /(c / a)=a / \lambda$, where $v$ and $c$ represent the frequency and speed of the light, becomes larger as $a$. Therefore, more modes are pushed above the light line of the air as $a$, which gives rise to the leakage of the light from the slab or the enhancement of the coupling with the radiation mode. Hence, PL intensity is enhanced as $a$ becomes longer. Such effect is experimentally observed by Ryu et al.[25,26]. We believe that our results are also explained in the simillar mechanism. The present discussion neglects the change of normalized pillar size $d / a$, which results in the modifictation of the photonic bandstructure, and the dependence of the light extraction efficiency on the parameters of PhCs is quite complicated[22]. Accurate numerical analysis on the light extraction efficiency is required and is left for future study. 


\subsection{High Aspect Ratio Nanowires}

In the sample shown in Figs. 1(b) and (c), the diameter $d$ and height $h$ of the pillar was about $0.24 \mu \mathrm{m}$ and $1.4 \mu \mathrm{m}$, giving aspect ratio of $h / d \sim 5.8$. As we described in Sec. 3.1, the height of the pillars are much larger than the thickness of the GaAs layer grown on reference planar (001) and (111)B substrates $(\sim 0.19 \mu \mathrm{m})$. Furthermore, the height becomes larger for pillars with smaller $d$ [16].

In order to use our pillars to the nanowires, it is necessary to grow the pillars with much smaller size and much higher aspect ratio. For this purpose, we prepare the mask with smaller $d_{0}$ by reducing the size of the initial patterning and dose of the electron beam. At the same time, we lengthen the growth time by a factor of 3 . The result of SAMOVPE is shown in Fig. 5(a). GaAs pillars exceeding aspect ratio of $100(d \sim 60 \mathrm{~nm}$ and $h>6 \mu \mathrm{m}$ ) were grown partly on the (111)B masked substrates. Similar nanowires are also grown with AlGaAs as shown in Fig. 5 (b). In spite of such small size, it is noted that the cross section of the pillar remains hexagonal. From these results, it is concluded that we can form nanowires with extremely small diameter and high aspect ratio and their arrays by SA-MOVPE.

\section{Summary}

We have demonstrated the formation of hexagonal GaAs/AlGaAs pillars by selective area metalorganic vapor phase epitaxial growth on (111)B GaAs masked substrates. Extremely uniform arrays of hexagonal pillars in triangular lattice have been obtained at optimized growth conditions. Photoluminescence study of GaAs/AlGaAs pillar array

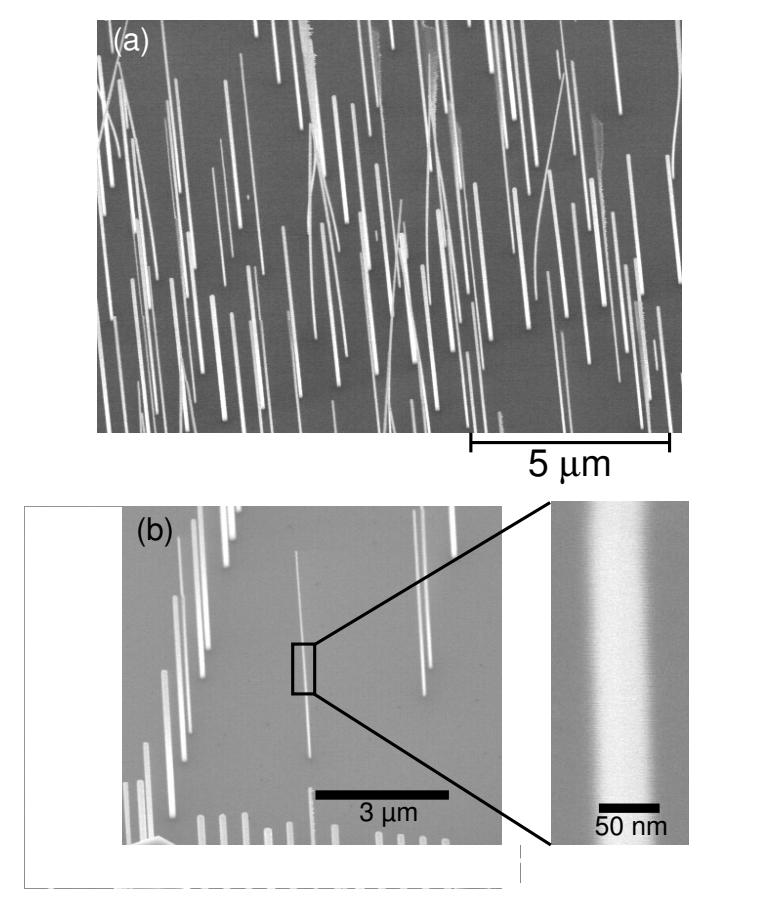

Fig. 5. SEM image of (a) GaAs and (b) AlGaAs/GaAs hexagonal pillars with extremely high aspect ratio.

strongly suggests the effect of light extraction efficiency in photonic crystals.

Our formation method of the pillars does not rely on the vapor-liquid-solid mechanism where metal (e.g. Au) is used as a catalyst to form as such GaAs and InAs nanowires reported so far. Therefore, it is expected to offer superior crystalline quality as well as good controllability of the growth to form heterostructured nanowires.

\section{Acknowledgement}

The authors thank Dr. M. Akabori for the idea of nanowire applications of selectivelygrown structures. They also acknowledge Prof. H. Hasegawa for stimulating discussions, and Mr. S. Akamatsu for supporting the experiment. This work was financially supported in part by a Grant-in-aid for Scientific Research supported by the Japan 
Society for the Promotion of Science.

\section{References}

[1] T. Fukui, S. Ando, Y. Tokura, and T. Toriyama, Appl. Phys. Lett. 58, (1991) 2018.

[2] S. Ando, N. Kobayashi, and H. Ando, Jpn. J. Appl. Phys. 32 (1993) L1293.

[3] P. Mohan, F. Nakajima, M. Akabori, J. Motohisa, and T. Fukui, Appl. Phys. Lett. 83 (2003) 689.

[4] M. Akabori, J. Takeda, J. Motohisa, and T. Fukui, Physica E 13 (2002) 446.

[5] J. Takeda, M. Akabori, J. Motohisa, and T. Fukui, Appl. Surf. Sci. 190 (2002) 236.

[6] M. Akabori, J. Motohisa, and T. Fukui, IEEE Conf. Proc. 27th Int. Symp. Compound Semiconductors, 191 (2001); M. Akabori, J. Takeda, J. Motohisa, and T. Fukui, Nanotechnology 14 (2003) 1071.

[7] M. Inari, J. Takeda, J. Motohisa and T. Fukui, in workbook of the 11th international conference of Modulated Semiconductor Structures (MSS-11) (Nara, Japan, 2003); M. Inari, J. Takeda, J. Motohisa and T. Fukui, accepted for publication in Physica E.

[8] T. Hamano, H. Hirayama, and Y. Aoyagi, Jpn. J. Appl. Phys. 36 (1997) L286.

[9] Y. Huang, X. Duan, Y. Cui, L. J. Lauhon, K.H. Kim, C. M. Lieber, Science 294 (2001) 1313.

[10] M. H. Huang, S. Mao, H. Fick, H. Yan, Y. $\mathrm{Wu}, \mathrm{H}$. Kind, E. Weber, R. Russo, and P. Yang, Science 292 (2001) 1897.

[11] J. Wang, M. S. Gudiksen, X. Duan, Y. Cui, and C. M. Lieber, Science 293 (2001) 1455
[12] K. Hiruma, H. Murakoshi, M. Yazawa, T. Katsuyama, J. Cryst. Growth 163 (1996) 231

[13] M. T. Björk, B. J. Ohlsson, T. Sass, A. I. Persson, C. Thelander, M. H. Magnusson, K. Deppert, L. R. Wallenberg, and L. Samuelson, Appl. Phys. Lett. 80 (2002) 1058.

[14] M. S. Gudiksen, L. J. Lauhon, J. Wang, D. C. Smith, and C. M. Lieber, Nature 415 (2002) 617

[15] K. Hiruma, M. Yazawa, T. Katsuyama, K. Ogawa, K. Haraguchi, M. Koguchi, and H. Kakibayashi, J. Appl. Phys. 77 (1995) 447.

[16] J. Noborisaka, J. Motohisa, and T. Fukui, unpublished.

[17] V. Malyarchuk, J. W. Tomm, V. Talalaev, Ch. Linau, F. Rinner, and M. Baeumler, Appl. Phys. Lett. 81 (2002) 346.

[18] S. S. Chang, S. Ando, and T. Fukui, Surf. Sci. 267 (1992) 214.

[19] S. Ando, N. Koybayashi, and H. Ando, Jpn. J. Appl. Phys. 37 (1998) L105.

[20] J. M. Moison, K. Elcess, F. Houzay, J. Y. Marzin, J. M. Gèrard, F. Barthe, and M. Bensoussan, Phys. Rev. B 41 (1990) 12945.

[21] S. Kodama, S. Koyanagi, T. Hashizume, and H. Hasegawa, J. Vac. Sci. and Technol. B 13 (1995) 1794.

[22] S. Fan, P. R. Villeneuve, J. D Joannopoulos, and E.F. Schubert, Phys. Rev. Lett. 78 (1997) 3294.

[23] T. Baba, K. Inoshita, H. Tanaka, J. Yonekura, M. Ariga, A. Matsutani, T. Miyamoto, F. Koyama, and K. Iga, J. Lightwave Technol. 17 (1999) 2113.

[24] M. Boroditsky, T. F. Krauss, R Coccioil, R. Vrijen, R. Bhat, and E. Yablonovitch, Appl. Phys. Lett. 75 (1999) 1036.

[25] H. Y. Ryu, Y. H. Lee, R. L. Sellin, and D. Bimberg, Appl. Phys. Lett. 79 (2001) 3573. 
[26] H.-Y. Ryu, J.-K. Hwang, Y.-J. Lee, and Y.-

H. Lee, IEEE Selected Topics on Quant.

Electron. 8 (2002) 231 